\title{
A Regional Model for E-Participation in the EU: Evaluation and Lessons Learned from VoicE
}

\author{
Sabrina Scherer and Maria A. Wimmer \\ University of Koblenz-Landau, Institute for IS Research, \\ Research Group e-Government, \\ Universitätsstraße 1, 56070 Koblenz, Germany \\ \{scherer, wimmer\} @uni-koblenz.de
}

\begin{abstract}
Attracting and motivating citizens to participate actively in online discussions of European policies turns out not to be easy. In the VoicE project, a regional e-participation model has been developed to deal with this challenge. The results and lessons learned from the project VoicE are of particular importance for the follow-up project VoiceS. This way, VoicE and VoiceS incorporate ongoing evaluation through an iterative design cycle. In this paper, we present the scientific evaluation method and results whether the regional eparticipation model of VoicE is an appropriate means to attract citizens for European policies and to motivate citizens to participate in discussions. The methodology is based on a layered model of e-participation evaluation. Subsequently, this paper examines to what degree the approach chosen in the project delivers suitable insights for establishing successful e-participation platforms on a European level and what lessons can be learned.
\end{abstract}

Keywords: E-Participation, Evaluation, E-Consultation.

\section{Introduction}

The VoicE $^{1}$ project is designed as a trial project, implementing a regional model of eparticipation in the European Union (EU), which places a high emphasis on platform marketing, editorial preparation and integration into the surrounding political institutions [1]. In this regard, VoicE provides two regional platforms ${ }^{2}$ serving as interfaces between decision-makers in the EU and citizens in the regional contexts. In terms of contents, the project focuses on the policy field of consumer protection. On both platforms, general information on the topics, a news section, polling functionality ("Question of the Month") and a discussion forum ("civil forum") are included. For the distribution of content also RSS feeds, Twitter messages, social bookmarking and newsletters are used. Texts are available in German on the BadenWürttemberg and in Spanish and Valencian on the Valencia instance. Fig. 1 shows a screenshot of the German platform. The feasibility of such an approach is of

\footnotetext{
${ }^{1}$ VoicE- Giving European people a voice in EU legislation, www . give-your-voice.eu

${ }^{2}$ Baden Württemberg, Germany (http://www.bw-voice.eu) and Valencia, Spain (http: / /www.voice.gva.es). Platform functionalities are described e.g. in [7].
} 
particular importance, as the follow-up project VoiceS ${ }^{3}$ continues the aims of the VoicE project (which was finished in December, 2009) and complements the platform by adding a series of new features such as a serious game and semantics [2]. This way, VoicE and VoiceS incorporate ongoing evaluation (as recommended by [3]) in an iterative design cycle [4].

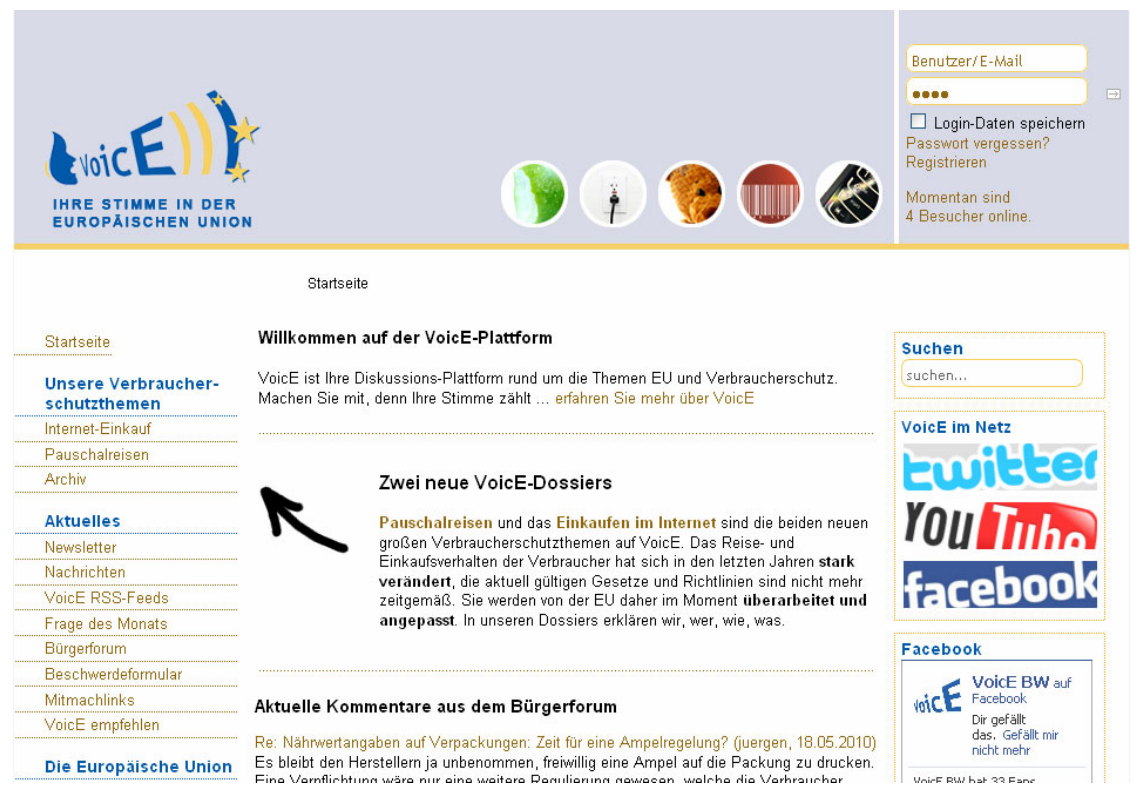

Fig. 1. Screenshot of www.bw-voice.eu (accessed in May, 2010)

This paper examines to what degree the approach chosen in VoicE delivers suitable tools for establishing successful e-participation platforms on a European level and what lessons can be learned for VoiceS. Section 2 introduces the evaluation methodology applied. Section 3 presents the evaluation results. Section 4 summarises lessons learned from the VoicE project. Section 5 gives a conclusion and an outlook on resulting activities for VoiceS to take lessons learned into consideration.

\section{Evaluation Methodology}

Evaluation of the VoicE project aims to assess if the regional e-participation model of VoicE is an appropriate means to attract citizens for European policies and to motivate them to participate in discussions. Fig. 2 further details this research question into three ones. The methodology applied to answer these questions is based on the layered model of e-participation evaluation of Macintosh and Whyte [5]. It integrates the project, socio-technical and democratic perspective on an

${ }^{3}$ VoiceS - Integrating Semantics, Social Software and Serious Games into eParticipation, www. eu-voices.eu 
e-participation exercise. Fig. 2 shows how the evaluation perspectives of [5] feed into the individual research questions formulated to evaluate VoicE.

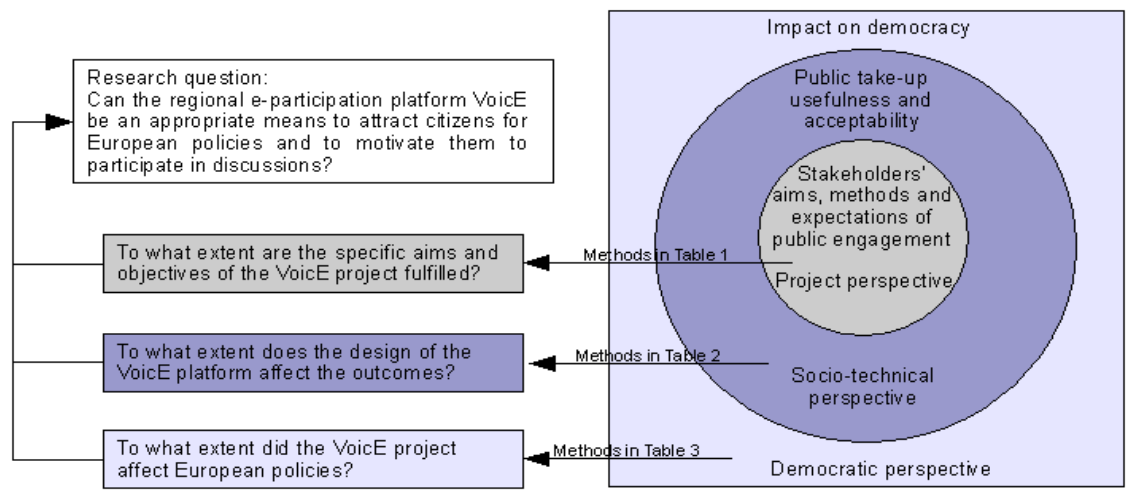

Fig. 2. Application of layered evaluation framework (based on [5])

Evaluation of an e-participation project should be performed at different stages: in the design and planning phase, in the implementation phase, and focusing on the final results [6]. At the same time, different stakeholders should be involved and different methods used [5]. In this respect, the evaluation methodology applied in VoicE bases on a number of results from applying different methods during the VoicE and VoiceS project life cycles:

\section{Planning and implementation phase of VoicE:}

1. Requirements for the VoicE system were based on surveys with citizens and politicians. $^{4}$

2. Field observations were collected with different platform versions [7].

3. Website statistics were tracked since the launch of the pilots. ${ }^{5}$

II. Requirements analysis in VoiceS:

4. Requirements were formulated for Voices ${ }^{6}$.

5. Analysis of legislative procedures in the $\mathrm{EU}^{6}$ was performed.

III. Evaluation phase of VoicE:

6. An online end user evaluation questionnaire was distributed among citizens to gather information about their perception of the VoicE platform (January November 2009 and filled out by 21 users). It was also envisaged to ask MEPs for their perception, but only one MEP participated in the survey. ${ }^{5}$

7. A survey undertaken in July/August 2009 among visitors of the German VoicE platform investigated, how web 2.0 contents and features are accepted in general

\footnotetext{
${ }^{4}$ VoicE Deliverable D2.1. End-users' requirements report. Internal $(5,2008)$.

${ }^{5}$ VoicE Deliverable D6.6 Evaluation report, including lessons learned. Internal (1, 2010).

${ }^{6}$ VoiceS Deliverable D2.1 Requirements analysis report: Specification of (user) requirements for the VoiceS platform with focus on process models and user roles. Internal $(5,2009)$.
} 
by the users and if the use of such features has an added value to the project. The online questionnaire was filled out by 164 respondents $^{7}$.

8. Surveys with experts on e-participation were conducted in the frame of the MOMENTUM $^{8}$ project with the aim to evaluate VoicE [8]: Answers from three experts were provided anonymously to the VoicE team. ${ }^{5}$

9. Analysis of the quantity and quality of users' contributions were based on the approach of Märker [9]. ${ }^{5}$

10. Polls on bw-voice.eu and voice.gva.es were asking users in December 2009 for their perception regarding their link to European politics. 18 answers were recorded on bw-voice.eu and 61 on voice.gva.es. ${ }^{5}$

The DEMO-net evaluation framework proposes a number of criteria for evaluating the perspectives. It provides indicators and measures as well as methods to get the data [6].

Table 1, Table 2 and Table 3 list the evaluation criteria and respective methods applied in the VoicE evaluation as described before (numbers for the methods indicate the methods referred to in the list above).

Table 1. Evaluation criteria and methods for project perspective

\begin{tabular}{|l|l|}
\hline Criteria & Applied methods \\
\hline Engaging with a wider audience & 3,10, Media review, evaluator assessment \\
\hline Obtaining better-informed opinions & $6,8,9$, evaluator assessment \\
\hline Scope of deliberation & 9 \\
\hline Effectiveness & Estimation of time spend \\
\hline Feedback & $6,9,10$ \\
\hline Process quality & 6,8, desk research \\
\hline Sustainability & $6,7,8$ \\
\hline
\end{tabular}

Table 2. Evaluation criteria and methods for socio-technical perspective

\begin{tabular}{|l|l|}
\hline Criteria & Applied methods \\
\hline Social acceptability & $\begin{array}{l}1,6,7,8, \text { evaluator assessment, analysis of system } \\
\text { concept }\end{array}$ \\
\hline Usefulness & $1,2,4,6,7,8$, evaluator assessment, website analysis \\
\hline Usability & $2,5,6$, website analysis, evaluator assessment \\
\hline
\end{tabular}

Evaluation results derived are presented in the subsequent section. Quantitative and qualitative results are not presented in detail for lack of space. Instead, we refer to the papers and deliverables mentioned above for more details.

7 VoicE report: Bedeutung und Gegenstand von Web 2.0 Technologien im Rahmen von bestehenden E-Partizipations-Projekten der Europäischen Kommission und besondere Analyse dieser Technologien im Bezug auf das Projekt VoicE/VoiceS (8, 2009).

${ }^{8}$ MOMENTUM - Monitoring, Coordinating and Promoting the European Union eParticipation Projects and Initiatives, www. ep-momentum. eu 
Table 3. Evaluation criteria and methods for democratic perspective

\begin{tabular}{|l|l|}
\hline Criteria & Applied methods \\
\hline Representation & $4,5,8$, evaluator assessment, desk research \\
\hline Engagement & $6,7,8,3,9,10$, website analysis \\
\hline Transparency & $6,7,8,9$ \\
\hline Conflict and consensus & 9, interview with moderators, website analysis \\
\hline Political equality & 8,3 \\
\hline Community control & $5,6,7,8$ \\
\hline
\end{tabular}

\section{Evaluation Results}

In section 3.1 general results and outcomes are introduced. The evaluation results, which are focusing on project, socio-technical and democratic perspectives, are presented in sections 3.2, 3.3 and 3.4.

\subsection{General Results and Outcomes}

The evaluation of numbers of visits and unique visitors show that - as a result of extensive dissemination activities - the VoicE platform was widely recognised. Yet, the website statistics also show that the majority of users only visit the platforms short-time. To get a basic idea of how VoicE services are accepted, the functionality and frequency of use is briefly described subsequently. The participants from the survey in method 7 were asked, which feature of the Internet platform they used or intended to use. Table 4 shows the corresponding figures.

Table 4. VoicE platform functions and their (considered) usage $(\mathrm{N}=164)$

\begin{tabular}{|l|l|l|l|l|}
\hline Service & Yes. & No. & I am not sure. & I do not know. \\
\hline Question of the month & $\mathbf{5 1 . 8 \%}$ & $23.8 \%$ & $12.8 \%$ & $11.6 \%$ \\
\hline Forum & $27.4 \%$ & $\mathbf{3 4 . 1} \%$ & $28.7 \%$ & $9.8 \%$ \\
\hline Read news & $\mathbf{7 3 . 8 \%}$ & $12.8 \%$ & $7.9 \%$ & $5.5 \%$ \\
\hline Subscribe newsletter & $22.6 \%$ & $\mathbf{5 3 . 0 \%}$ & $17.7 \%$ & $6.7 \%$ \\
\hline RSS-Service & $7.9 \%$ & $36.6 \%$ & $16.5 \%$ & $\mathbf{3 9 . 0 \%}$ \\
\hline Search & $\mathbf{5 1 . 8 \%}$ & $21.3 \%$ & $15.9 \%$ & $11.0 \%$ \\
\hline Twitter & $7.9 \%$ & $\mathbf{4 9 . 4 \%}$ & $20.7 \%$ & $22.0 \%$ \\
\hline Social bookmarking & $11.0 \%$ & $\mathbf{5 1 . 2 \%}$ & $22.0 \%$ & $15.9 \%$ \\
\hline Information provision & $\mathbf{5 0 . 6 \%}$ & $21.3 \%$ & $19.5 \%$ & $8.5 \%$ \\
\hline
\end{tabular}

The "Questions of the Month" are succinct polls that change on the platform in a monthly frequency to collect citizens' opinions on specific questions. Often, these are used to put forward a question in the name of a politician. Such questions are mainly of type yes/no answers. In some cases, a third possibility in the form of "I do not know" or "I did not think about it" is inserted. The trend of users' participation is increasing in both regions. On average, about 90 users answer the questions per month. 
The citizens' forum is a discussion area where users can write their opinions and comments and exchange their views. It is divided into the various discussion topics (toy safety, food, energy, market watch, and telecommunication), and "general topics". Participation in the forum is possible for unregistered users since summer 2009. The majority of threads is viewed between 1,000 and several thousand times. Based on the frequency of views, an importance for the Internet platform and attention of the user can be attested. However, the active participation is too low in comparison to page visits. VoicE therefore needs to intensify its work to find proper participation possibilities, which are noticed by both, citizens and politicians. With the "Letter to Brussels" initiatives, MEPs should be directly informed about the opinions of their voters from the regions. It was planned that MEPs formulate a reply and send this to the VoicE team (for publication on the website) or discuss in the forum. One problem is that few reactions of the politicians discourage citizens to invest their time in further discussions. Vice versa, input of only few citizens discourage politicians to invest their time in reactions. Despite rather low participation of citizens and political stakeholders, the "Letter to Brussels" initiative gives an idea and impression for further activities. It should also be noticed that such an initiative needs to be established to increase the number of participants (on both sides - citizens and politicians).

\subsection{Project Perspective}

The analysis is focussing on the specific aims and objectives of the project.

Engaging with a wider audience: VoicE contributes in two ways to engage people: informing about participation possibilities and motivating people to participate first time. The majority of platform visitors is from Baden-Württemberg and Valencia respectively. This is due to the fact that most dissemination activities are performed in these regions. Strong efforts are made to promote the platforms using different online and offline channels. Online marketing (newsletters, emails etc.) turned out much more useful and effective than offline marketing. The target groups (citizens in both regions) are very diverse in age, social background, interests etc. However, the social profile of involved people could not be analysed because such data are not available? In consequence, dissemination material produced and activities performed as well as the look-and-feel of the platform were not customised for a specific age or social group. It seems that this decision negatively influenced the attractiveness of VoicE. In general, individual participation and networking on both platforms was rather low. Concentration and focus on a smaller target group might be considered in the future.

Obtaining better informed decisions: Studies show that the majority of European citizens has little knowledge about the EU, its institutions and its functions. They also underline that a number of citizens do not have an idea of the EU's influence on national legislation $[10,11]$. The VoicE platform aims to inform users about such issues. An evaluator assessed the website contents as texts being comprehensive and in general easily understandable, but too long. The last issue rather deters users from reading. In contrast, news on the website are rather short and up-to-date, sometimes

\footnotetext{
${ }^{9}$ Registration is not necessary to actively participate in the VoiceE platform.
} 
linked with the forum and provide important background information about the topics. In general, a high information factor of the platform was underlined by 20 of 21 respondents of the end user evaluation questionnaire. They agreed that the platform provides proper tools to sufficiently inform them about the topics under discussion. Hence it can be stated that VoicE enhances the scope of expertise of informing citizens.

Scope of deliberation: The interactivity among the participants of the citizens' forum is rather little and most comments are rather short, consisting of 3 or 4 sentences. Almost all start entries originate from moderators. One positive aspect is that in the majority of cases, the work of the moderators focused on explanations with regards to content, summarisations, or posting motivating questions.

Most comments keep the issue of discussion and reasonably deal with it. Only in few cases, postings were obviously malevolent or nonsense. The overall majority of comments refers to individual experience and what might be called common sense. These comments are not built upon inter-subjectively available data and stringent argumentative figures. Thus, the discussion consists more or less of individual positions put opposite each other or underline a position (as already explained in other evaluations, see [12]). Nevertheless, it was manageable to summarise the discussions and derive a common final statement. The platform does not provide an argumentation visualisation of options and no technical support to summarise comments.

Effectiveness: Efforts spent in VoicE cannot be evaluated against offline processes because there was no comparable initiative. It is not possible to compare saved costs and time. The only figures, which can be evaluated, are average hours spent per week for moderation of discussions, updating information, posting news, and general efforts to keep the platform alive. The figures are similar on both platforms, whereas the effort for moderation is a little higher on one platform caused in the fact that more discussions took place there. Most effort is spent to search and post news to keep the platforms up-to-date. When comparing traditional with electronic participation opportunities, lack of knowledge of people in such services needs to be noted. Addressing the effectiveness from a user perspective, the project could not achieve the objective that users' voice was really heard (see next point). Time spent by users seem to be rather low, as website statistics show.

Feedback: Feedback of politicians was rather low and unsatisfying for the users. Only one MEP answered two times in the bw-voice.eu forum. No other reactions were recognised on the different letters, which the organisers from Valencia and BW sent to MEPs. The politician who answered the evaluation questionnaire stressed the lack of time as reason for not participating in the discussions. In this regard, the offered participation processes are not sufficiently transparent. It does not become clear to the user, which information or decisions of the ongoing process are available and where impact or at least influence can be achieved. This is caused in the fact that consulted MEPs do not provide a feedback. Indeed, the VoicE platform visualises the EU decision making process, even though it might be helpful to provide a simpler version of the process focussing on the explanations how the contributions feed into the process. Explicit information on expectable feedback should be provided to participants on their inputs. This could not be provided in VoicE because it was not 
clear what MEPs do and how they react on the citizens' opinions forwarded by the project. Here a stronger adaption on political processes is necessary.

Process quality: The design process of the VoicE portal was an iterative and welldefined process. It was influenced by the heuristic analysis performed by project partners and the empirical testing with pilot users. It turned out useful to improve the system [7]. This usability engineering process has been approved by the results of the evaluation regarding usefulness and usability of the system (see section 3.3).

The quality of the participation processes was evaluated by analysing its appropriateness, appeal and economics. The objectives of the participation processes should be better communicated to target groups. It should also be pointed out what happens with the discussions and how users can participate in the legislative process. The participation processes need to be analysed for their appropriateness to the topic.

Sustainability: In this regard, the respondents of the end user evaluation questionnaire (see method 6) were asked if they would continue using the VoicE platform. The question if the topics discussed attract them to return to the platform was positively answered by all 21 respondents. The question of the "visit frequency and continuity" was also part of the questionnaire for the analysis of web 2.0 tools (see method 7) for e-participation. A "return" to VoicE $(\mathrm{N}=164)$ is intended by $75.4 \%$ of the one time visitors, $95.2 \%$ of the two times visitors, $90 \%$ of three or four time visitors and $100 \%$ of 5 times or more visitors. In summary, the data show a positive signal for the sustainability of VoicE regarding VoiceS if weak points are eliminated. In particular the consumer protection topics discussed would attract users to return to the platform.

\subsection{Socio-Technical Perspective}

This evaluation perspective investigates the usefulness, usage and acceptability of the platform regarding users and processes.

Social acceptability: It can be stated that information presented is accurate, complete and reliable. Privacy of information is performed by the controlled access to information. The user's password is used for the authentication mechanisms. To participate in the forum and in online, the user does not need to register, but needs to provide email and user name. The email is not visible to other users. The data necessary for registration in the platform are minimal (user name, e-mail, password).

Usefulness: Commonly used platform functions include reading news and information provision offerings. Polls are the only form of active participation, which is frequently used by the participants. Social bookmarks and Twitter are rather unused. Participation in the citizens' forum is a core component of the possibility of active participation in VoicE but rather not used in this form.

The user surveys (see methods 6 and 7) show that users do not miss any specific functionalities and services, which they may know from other participation experiences. But experts recommend the usage of further communication and community building functionalities. It was e.g. proposed by one expert to consider a kind of petitioning tool instead of or in addition to aggregating discussion results by a moderator. It can be concluded that tools provided are useful and appropriate for this 
kind of participation. Experts recommended making the tools more interactive. Resulting from the low number of active participants in the platform and expert statements the participation processes should be reconsidered. In this context, the participation tools should be adapted.

Usability: A good usability was stated by survey respondents and experts even if there are some minor usability flaws, which should be eliminated. The technologies and tools deployed are perceived rather accessible for all (because "essential parts of the content are available in audio format") even if not according to WCAG standards. In general, the usability of the VoicE platform resulted from a comprehensive usability engineering process.

\subsection{Democratic Perspective}

This perspective aims to understand to what extent the VoicE affects the democratic goals.

Representation: Provided information is correct, comprehensive and easily understandable (cf. section 3.2) and therefore enhances the understanding in this form of representative democracy. This is of significant importance as the general knowledge in the EU is rather low (see e.g. [10,11]). VoicE aimed to enhance especially the role of MEPs by making it more visible and by offering citizens the chance to give their opinions on recent activities in the EU. The low support from the side of European politicians hindered this.

Engagement: The objectives of the project address the level of engagement in informing and consultation. While the first was fully reached, the second was only partly reached. Information on (electronic) participation possibilities is rather rare. One example is the petitioning procedure, which is explained on the Valencia platform but not yet on the German one. The same is the case with knowledge about existing initiatives e.g. running consultations of the EC, which is rather sparse on both platforms. General willingness of users to actively participate in political topics is rather low, even if they are interested in the topic. For those persons who are willing to actively participate, VoicE contributed partly to this decision. But the attractiveness and participation possibilities should be improved to engage more visitors. In conclusion to this, VoicE is rather used as information than as participation offering.

Transparency: The VoicE pilot has increased transparency of EU politics partly by providing simple explanations of the EU legislative procedures, the institutions and consumer protection issues as well as by publishing the latest news on these issues. A strength of VoicE in this regard is the dynamic tool showing progress of legislation process. This should be even more brought to the fore and used to explain the legislative process in general in an interactive way. The possibilities for influencing the decision-making process were not yet made sufficiently transparent.

Conflict and consensus: The VoicE platform supports the divergence of opinions and the deliberation about a topic in the forum. In general, the level of agreement in the discussions is rather high. But there are also deliberations about pros and cons of issues among the participants. The moderators need to manage the diversity when summarising the discussions e.g. for a "Letter to Brussels". In this context, it should 
be considered, as recommended by the experts, to use a kind of petition tool and provide the opportunity for the users to summarise their opinions themselves.

Political equality: The project shows strong potential for greater inclusiveness. There were signs that participants were mostly not previously "engaged" in political decision making. But there are no numbers available about the political, ethical, cultural, social etc. variety of users.

Community control: Most users are rather unsatisfied with the influence they reached in the legislative process. They do not think that - or are not sure if - their contributions will be further considered. The majority of these respondents did not expect higher achievements and they see the citizens' forum, polls and letter to Brussels to be of low to medium importance for the politicians. More efforts need to be made to improve participation rates by e.g. facilitating electronic debates, fostering participation on the same topics offline, making the final impact clear. Citizen engagement also needs to be linked appropriately to decision making processes.

\section{Lessons Learned}

The lessons learned are provided along six topics:

Regional approach: The question if regional e-participation is an appropriate means to interest citizens for European policies and motivate them to participate was analysed. Analysis evidences that the regional approach attracts citizens to visit the website and in some cases also to participate. A number of platform visitors from the regions confirm that they have now a better or slightly better link to European politics. The regional focus helps citizens to recognise the direct effects of European politics on their own life. Therefore the EU comes closer to the citizens. However, as the provision of participation opportunities on EU level is rather low (and often has no real impact on the decision-making process), it is a hard task to reach an impact with citizens' participation. Hence, a regional e-participation model towards the EU is supportive to attract citizens' interest. Yet to have a voice in Brussels, citizen participation must be linked directly with decision-making at Parliament level.

Heterogeneous target groups: As VoicE's target group is quite diverse and because a one-approach-for-all strategy was decided, it was difficult to reach many people of different age groups and with different (political) interests with no particular address. A rather serious look-and-feel does not attract users who are familiar with web 2.0 websites as e.g. younger citizens. On the other side, another look could deter older citizens from visiting the platform. The same can be assessed for the topics discussed. The biggest flaws in unmotivated participation in political decision making resulted from the low impact of participation and the low participation of politicians on the platform.

Involvement of politicians could not be successfully implemented in VoicE. It was only possible to involve politicians superficially and only if no further work was requested from them (e.g. with the question of the month). Only one MEP felt up to react on user comments and a letter to Brussels with some posts in the forum. Politicians are overcharged with their usual work and a huge amount of participation possibilities that ask them to contribute. 
Relevance of the topic: The topic of discussion is crucial for an e-participation initiative. In VoicE the users were to a lesser extent interested in European politics than in consumer protection topics. It is important that participants are directly concerned by the topic selected.

Use of technologies: The use of each technology needs to be seen in the overall participation context. Tools used need not only to be adapted to the user requirements but also to the participation processes. The use of web 2.0 technologies does not ensure high user participation regardless how nice and easy to use they are.

Participation processes: It is key for a successful e-participation initiative that there are well-defined participative processes and that outcomes have an impact. In VoicE, this could not be achieved because politicians did not include users' contributions in their decision making processes. Other participation opportunities should be envisaged.

\section{Conclusion and Outlook}

In this contribution we investigated whether the VoicE model of regional eparticipation is suitable to attract users for European politics. The analysis evidenced that regionalised information and up-to-date news provide a proper means to inform and attract citizens. Most platform visitors appreciated the information offerings. Even though the wider target group is rather not interested in active participation, some visitors discussed EU legislation and the impact on the VoicE platform. Some challenges of wider citizen participation lay in the need for targeted tools for older vs. younger citizens. Likewise, interests in political topics vary among different ages. A future version of the VoicE platform should become more interactive. If participation of citizens in EU politics shall be successful, e-participation initiatives need to achieve an impact. This means that participation processes need to be aligned to show how citizens' contributions will be further considered in the decision making processes. Likewise, politicians and citizens need to get into direct dialogue, i.e. also politicians need to be ready to interact with the citizenship. E-participation initiatives such as the VoicE project need therefore to be linked up with the processes provided by the European Union (e.g. running consultations or petitions from the European Parliament).

The VoicE consortium can address these points in VoiceS. Resulting from the evaluation, a number of activities are envisaged in the VoiceS project:

1. Promotion activities and the look-and-feel of the website should be focused to specific conditions of the diverse target groups. In VoiceS, it is envisaged to concentrate activities to the specific target group of younger citizens.

2. Involvement of MEPs and other politicians should be triggered with a welldefined participation process going along with media coverage.

3. Information texts should be shortened and elaborated for the targeted group.

4. The participation processes should be made more transparent.

5. The platform should get more interactive. The discussion forum should be replaced by a comment functionality. 
Acknowledgment. VoicE and VoiceS are co-funded by the European Commission under the eParticipation Preparatory Action. This publication reflects the views only of the authors, and the Commission cannot be held responsible for any use which may be made of the information contained therein.

\section{References}

[1] Holzner, M., Schneider, C.: Consumer Protection, European Decision-Making and the Regions - the eParticipation Project VoicE. In: Cunningham, P., Cunningham, M. (eds.) Collaboration and the Knowledge Economy: Issues, Applications, Case Studies Part 1, pp. 351-356. IOS Press, Amsterdam (2008) ISBN 978158603924-0

[2] Scherer, S., Holzner, M., Karamagioli, E., Lorenz, M., Schepers, J., Wimmer, M.A.: Integrating Semantics, Social Software and Serious Games into eParticipation: The VoiceS Project. In: Tambouris, E., Macintosh, A. (eds.) Electronic Participation: Proceedings of Ongoing Research, General Development Issues and Projects of ePart 2009, pp. 151-158. Trauner Verlag, Linz (2009)

[3] Millard, J.: eParticipation recommendations - focusing on the European level. Deliverable 5.1 , Study and supply services on the development of eParticipation in the EU (July 2009)

[4] Scherer, S., Wimmer, M.A., Ventzke, S.: Hands-on guideline for e-participation initiatives. In: Proceedings of the E-Government and E-Services (EGES) conference 2010. IFIP AICT. Springer, Heidelberg (2010) (to appear in September 2010)

[5] Macintosh, A., Whyte, A.: Towards an evaluation framework for eParticipation. Transforming Government: People, Process and Policy 2(1), 16-30 (2008)

[6] Lippa, B., Aichholzer, G., Allhutter, D., Freschi, A.C., Macintosh, A., Westholm, H.: D 13.3: eParticipation Evaluation and Impact. DEMO-net (2008) (Deliverable)

[7] Scherer, S., Karamagioli, E., Titorencu, M., Schepers, J., Wimmer, M.A., Koulolias, V.: Usability Engineering in eParticipation. European Journal of ePractice (7) (2009)

[8] Bicking, M., Wimmer, M.A.: Evaluation framework to assess eParticipation projects in Europe. In: Tambouris, E., Macintosh, A. (eds.) Electronic Participation: Proceedings of Ongoing Research, General Development Issues and Projects of ePart 2009, Schriftenreihe Informatik, vol. 31, pp. 73-82. Trauner Verlag, Linz (2009)

[9] Märker, O.: Evaluation von E-Partizipation. In: E-Partizipation: Beteiligungsprojekte im Internet. Beiträge zur Demokratieentwicklung, vol. 21, pp. 252-281. Stiftung Mitarbeit, Bonn (2007)

[10] EUROBAROMETER: Public Opinion in the European Union. National Report, Executive Summary: Germany 70, Eurobarometer (2008)

[11] EUROBAROMETER: Public Opinion in the European Union. First Results 70, Eurobarometer (December 2008); Fieldwork (October-November 2008); Publication (December 2008)

[12] Scherer, S., Neuroth, C., Schefbeck, G., Wimmer, M.A.: Enabling eParticipation of the Youth in the Public Debate on Legislation in Austria: A Critical Reflection. In: Macintosh, A., Tambouris, E. (eds.) ePart 2009. LNCS, vol. 5694, pp. 151-162. Springer, Heidelberg (2009) 